\title{
Exact solution of unsteady MHD free convective flow with constant heat flux: revisited
}

\author{
Basant K. Jha, Luqman A. Azeez, Michael O. Oni
}

Department of Mathematics, Ahmadu Bello University, Zaria, Nigeria

\begin{tabular}{l} 
Article Info \\
\hline Article history: \\
Received Mar 29, 2019 \\
Revised Oct 22, 2019 \\
Accepted Mar 20, 2020 \\
\hline
\end{tabular}

\section{Keywords:}

Free convection

Heat flux

Impulsive motion

MHD

Riemann-sum approximation

\begin{abstract}
A solution of unstable MHD free convective flow of an incompressible, viscous and electrically steering fluid created by spontaneous motion of vertical plate subjected to constant heat fluctuation offered by Sacheti et al. has been revisited. The governing equations are deciphered with the aid of Laplace transform procedure, while the inversion is gotten through the Riemann sum approximation approach. Fluid velocity are varied with sundry parameters such as, Prandtl number, Grashof number and Hartmann number have been extensively explicated with the help of graphs. Numerical comparison is carried out and equated with the benchmark values reported in literature and an outstanding agreement is established. This article targeted at amending some discrepancies in the skin-friction offered by Sacheti et al. The benefit of the suggested technique could be a reduction of computation time.
\end{abstract}

This is an open access article under the CC BY-SA license.

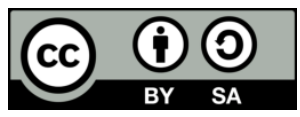

\section{Corresponding Author:}

Basant K. Jha

Department of Mathematics

Ahmadu Bello University

Com market, ABU, Zaria, Nigeria

Email: basant777@yahoo.co.uk

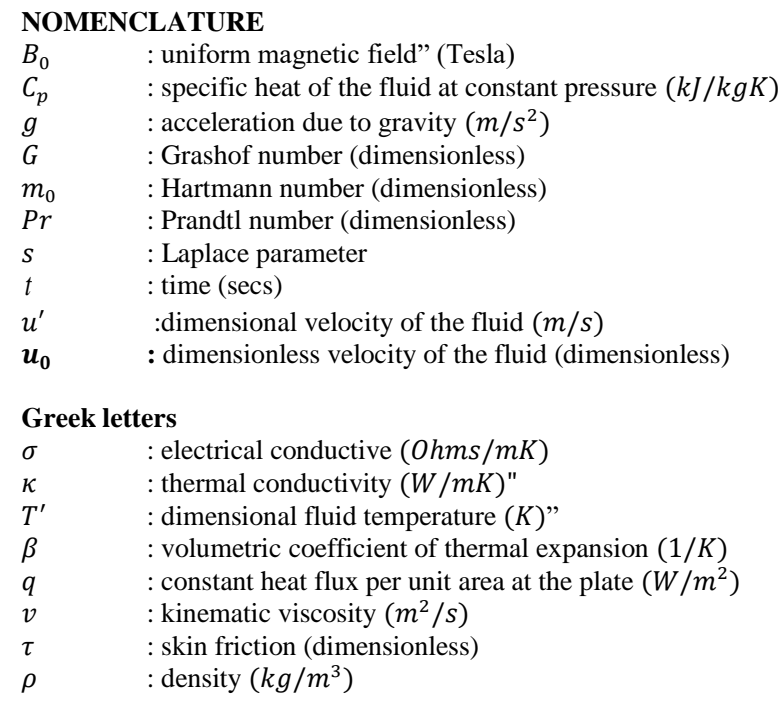




\section{INTRODUCTION}

The study of hydromagnetic convective flow has drawn serious attention over the decades based on its scientific and technological applications, such as; nuclear reactors, spacecraft design, geothermal energy extraction, boundary flow layer control, plasma studies, metallurgy, mineral, petroleum engineering, chemical and solar energy collectors. Numerous studies have been conveyed on magnetohydrodynamics flow with diverse physical conditions. Soundalgekar and Patil [1] gave analytical study on stokes problem for infinite vertical plate with constant heat flux. The unsteady hydromagnetic free convection flow with constant heat flux was considered by Sacheti et al. [2]. In their work, by using the method of convolution integration, they concluded that the magnetic field has a impeding effect on the velocity. Kafousias et al. [3] extended their finding by incorporating the role of the free-convection flow past an impulsively vertical porous plate with constant suction. Ibrahim et al. [4] investigated the influence "of chemical reaction and radiation absorption on the unsteady MHD free convection flow past a semi-infinite vertical permeable moving plate with heat source and suction". Also, Anand et al. [5] discussed the influence of radiation on an unsteady MHD free convective flow past a vertical porous plate in the presence of soret. Asma et al. [6] examined the unsteady MHD free convection flow of cassion fluid past over an oscillating vertical plate embedded in a porous medium while Israel et al. [7], further extended the work of [6] by investigating the role of "viscous dissipation and rotation on unsteady MHD free convection flow past an infinite heated vertical plate in a porous medium with time dependent suction". In another related work, Singh [8, 9] investigated the "MHD free-convection flow past an accelerated vertical porous plate in a rotating fluid and unsteady free convection flow through a rotating porous medium". The problem for a viscous incompressible and electrically conducting fluid in the presence of a transverse magnetic field was discussed by Nanousis et al. [10].

"Some recent works related to the present investigation are found in the literature [11-16]. Jha et al. [11] analytically studied the fully developed steady natural convection flow of conducting fluid in a vertical parallel plate microchannel in the presence of transverse magnetic field. The effect of Hartmann number was reported to decrease the volume flow rate. The combined influence of externally applied transverse magnetic field and suction/injection on steady natural convection flow of conducting fluid in a vertical microchannel was carried out by Jha et al. [12]. In another work, Jha et al. [13] examined the effect of wall surface curvature on transient MHD free convective flow in vertical micro-concentric-annuli. Jha et al. [14] studied exact solution of steady fully developed natural convection flow of viscous, incompressible, and electrically conducting fluid in a vertical annular microchannel. Recently, Jha and Aina [15] presented the magnetohydrodynamic (MHD) natural convection flow in a vertical micro-porous-annulus (MPA) in the presence of radial magnetic field. Also, the MHD natural convection flow in vertical micro-concentric-annuli (MCA) in the presence of radial magnetic field has been analyzed by Jha et al. [16]".

The objective of this article is to revisit the work of [2] by presenting a semi-analytical solution to the work. The numerical values displays that the present solution calculates the same fluid velocity as presented [2]. In addition, the physical justification and table presented for skin-friction by [2] is wrongly presented, and this makes their analytical result for skin-friction unreliable and incorrect. In view of this, we set out to give a correct expression for skin-friction and justification of result.

\section{MATHEMATICAL ANALYSIS}

Reexamine the mathematical equations offered by [2]. Following their equations and rules, the governing momentum and energy balance equations describing the current investigation in nondimensionless forms are [2]:

$$
\begin{aligned}
& \frac{\partial u^{\prime}}{\partial t^{\prime}}=v \frac{\partial^{2} u^{\prime}}{\partial{y^{\prime}}^{2}}+g \beta\left(T^{\prime}-T_{\infty}^{\prime}\right)-\frac{\sigma B_{0}^{2} u}{\rho} \\
& \frac{\partial T^{\prime}}{\partial t^{\prime}}=\frac{\kappa}{\rho c_{p}} \frac{\partial^{2} T^{\prime}}{\partial y^{\prime 2}}
\end{aligned}
$$

Subjected to the corresponding conditions:

$$
\begin{aligned}
& t^{\prime} \leq 0, u^{\prime}=0, T^{\prime}=T_{\infty}^{\prime} \text { for } y^{\prime} \geq 0 \\
& t^{\prime}>0\left\{\begin{array}{l}
u^{\prime}=u_{0}, \frac{\partial T^{\prime}}{\partial y^{\prime}}=-\frac{q}{\kappa}, \text { at } y^{\prime}=0 \\
u^{\prime} \rightarrow 0, T^{\prime} \rightarrow T_{\infty}^{\prime}, \text { as } y^{\prime} \rightarrow \infty
\end{array}\right.
\end{aligned}
$$


Presenting the ensuing dimensionless quantities as shown in (1)-(3)

$$
\left.\begin{array}{l}
y=\frac{u_{0} y^{\prime}}{v}, u=\frac{u^{\prime}}{u_{0}}, t=\frac{u_{0}{ }^{2} t^{\prime}}{v}, T=k u_{0} \frac{\left(T^{\prime}-T_{\infty}^{\prime}\right)}{(q v)} \\
\operatorname{Pr}=\frac{v \rho C_{p}}{k}, G=\frac{q g B_{0} v^{2}}{\left(k u_{0}{ }^{4}\right)}, m_{0}=\frac{\sigma B_{0}{ }^{2} v}{\left(\rho u_{0}{ }^{2}\right)}
\end{array}\right\}
$$

By (4), (1), and (2) are obtained in dimensionless form as [2]:

$$
\begin{aligned}
& \frac{\partial u}{\partial t}=\frac{\partial^{2} u}{\partial y^{2}}-m_{0} u+G T \\
& \frac{\partial T}{\partial t}=\operatorname{Pr} \frac{\partial^{2} T}{\partial y^{2}}
\end{aligned}
$$

Subject to

$$
\begin{aligned}
& t \leq 0, u=0, T=0, \text { for } y \geq 0 \\
& t>0\left\{\begin{array}{l}
u=1, \frac{\partial T}{\partial y}=-1, \text { at } y=0 \\
u^{\prime} \rightarrow 0, T \rightarrow 0, \text { as } y \rightarrow \infty
\end{array}\right.
\end{aligned}
$$

To decipher (5) with boundary conditions (7), the temperature profile is first obtained from (6) and constituted into (5). By the Laplace transform (LT), the exact solutions are acquired via the LT as follows:

$$
\begin{aligned}
& \bar{u}(y, s)=\int_{0}^{\infty} u(y, t) e^{-s t} d t \\
& \bar{T}(y, s)=\int_{0}^{\infty} T(y, t) e^{-s t} d t
\end{aligned}
$$

where $s>0$ and taking the LT of (5) and (6) with the initial and boundary conditions (7), as shown in (5) and (6) become:

$$
\begin{aligned}
& \frac{d^{2} \bar{u}}{d y^{2}}-(m+s) \bar{u}+G \bar{T}=0 \\
& \frac{d^{2} \bar{T}}{d y^{2}}-\operatorname{Pr} s \bar{T}=0
\end{aligned}
$$

Therefore, the solutions of (9) and (10) in Laplace domain are:

$$
\begin{aligned}
& \bar{u}=\frac{1}{s} \exp \left[-y \sqrt{\left(m_{0}+s\right)}\right]+\frac{G}{\left(s \operatorname{Pr}-m_{0}-s\right)} \frac{\left[\exp (-y \sqrt{s P r})-\exp \left[-y \sqrt{\left(m_{0}+s\right)}\right]\right]}{s \sqrt{s}} \\
& \bar{T}=\frac{1}{s \sqrt{s \operatorname{Pr}} \exp (-y \sqrt{s \operatorname{sr}})}
\end{aligned}
$$

To obtain the velocity and temperature distributions in the time domain, (11) and (12) are to be inverted, since these equations are difficult to be inverted in closed form, we use a numerical procedure used by [17] which based on the Riemann-sum approximation (RSA) method.

Laplace domain can be inverted to the time domain $t$ as follows:

$$
\begin{aligned}
& u(y, t)=\frac{e^{\epsilon t}}{t}\left[\frac{1}{2} \bar{u}(y, \epsilon)+\operatorname{Re} \sum_{k=1}^{N} \bar{u}\left(y, \in+\frac{i k \pi}{t}\right)(-1)^{k}\right] \\
& \theta(y, t)=\frac{e^{\epsilon t}}{t}\left[\frac{1}{2} \bar{\theta}(y, \epsilon)+\operatorname{Re} \sum_{k=1}^{N} \bar{\theta}\left(y, \epsilon+\frac{i k \pi}{t}\right)(-1)^{k}\right]
\end{aligned}
$$


where $N$ is the number of terms used in the Riemann-sum approximation (RSA), $\in$ is the real part of the Bromwich contour that is used in inverting Laplace transforms and $R e$ refers to the real part of is the imaginary number $i$. The RSA for the Laplace inversion involves a single summation for the numerical process. The correctness of this method depends on the value of $\in$ and its truncation error dictated by $N$. Based on numerical experimentation and according to Tzou [18], the value of $\in$ must be selected so that the Bromwich contour encompasses all the branch points. For quicker convergence, the quantity $t=4.7$ gives the most satisfactory results.

\section{"Skin-friction"}

The skin friction $(\tau)$ are gotten as:

$$
\tau=\left.\frac{d u}{d y}\right|_{y=0}=-\frac{\sqrt{m_{0}+s}}{s}+\frac{G\left(\sqrt{s P r}-\sqrt{m_{0}+s}\right)}{s \sqrt{s P r}\left(s \operatorname{Pr}-m_{0}-s\right)}
$$

The solutions obtained are in Laplace domain and are converted to time domain by applying the RSA stated as shown in (13) and (14).

\section{RESULTS AND DISCUSSION}

For validation of accuracy of RSA method, we employed MATLAB software to obtain numerical values of the dimensionless velocity and skin friction and compared our results with those presented by Sacheti et al. [2]. Figure 1 exhibits the role of non-dimensional time $(t)$ and Prandtl number $(P r)$ on velocity profile. It is clear from Figure 1 that, increasing $t$, leads to a corresponding increase in the fluid velocity for both Prandtl numbers $(P r=0.71)$ and $(P r=7.0)$ but, it is more prominent for the case of air. This is because increase in temperature increases the convection current so that velocity also increases with time.
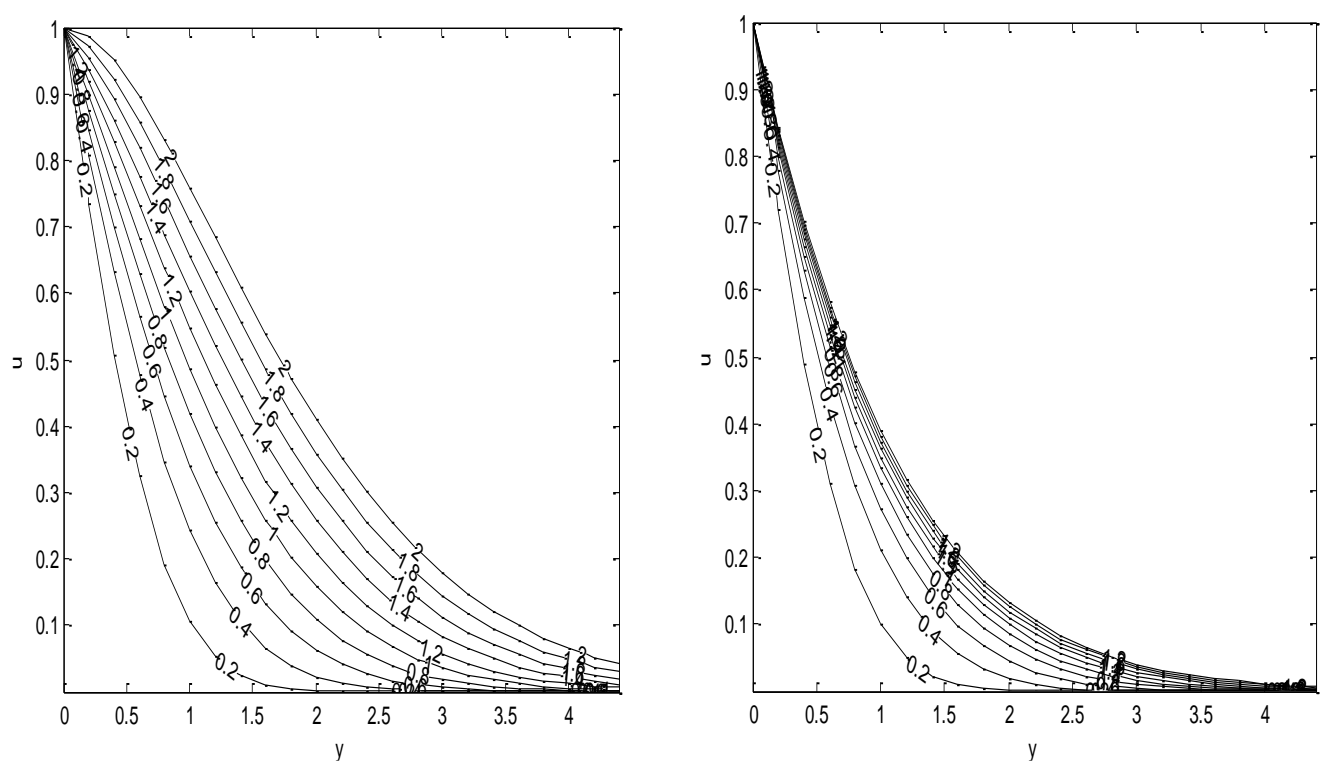

Figure 1: "Velocity profile showing the effect of $t$ for $\operatorname{Pr}=0.71$ and $\operatorname{Pr}=7.0$ " respectively, with fixed values of $\left(G r=1.0 m_{0}=0.5\right)$

Figure 2 illustrates the impact of Hartmann number $\left(m_{0}\right)$ and $\operatorname{Pr}$ on velocity profile. It is detected that for both Prandtl numbers $(P r=0.71)$ and $(P r=7.0)$ that, increase in magnetic field has a tendency to retard the motion of fluid flow in the vertical plate. This is because the application of magnetic field creates a resistive force similar to the drag force that acts in the opposite direction of the fluid motion, thus causing the fluid velocity to decreases. Figure 3 presents the role of buoyancy force on fluid velocity for different values of $(\mathrm{Pr}=0.71,7.0)$. It is realized that, fluid velocity increases as buoyancy force increases for both Prandtl numbers, but the significance effect is more noticeable in the case of air compare to water. 

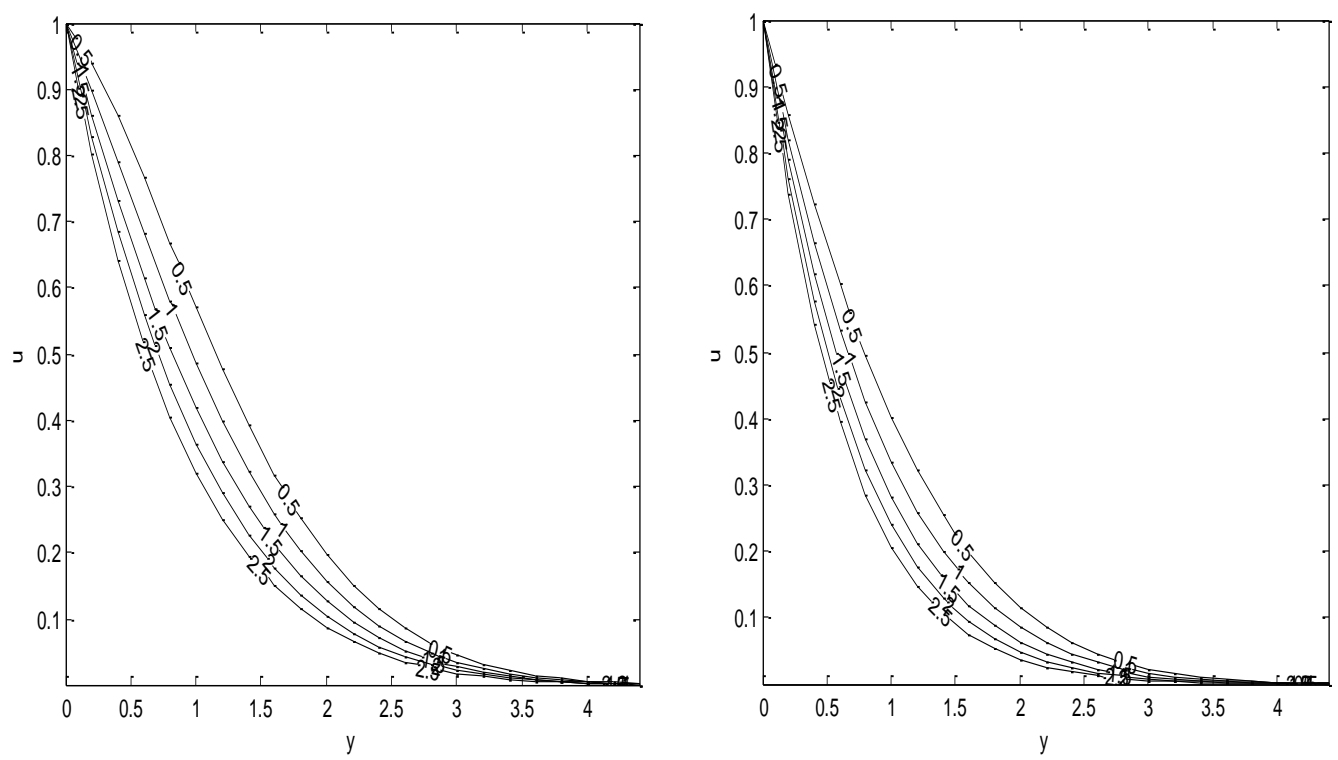

Figure 2. Velocity profile showing the effect of $m_{0}$ for $\operatorname{Pr}=0.71$ and $\operatorname{Pr}=7.0$ " respectively, with fixed values of $(t=1.0 \mathrm{Gr}=1.0)$
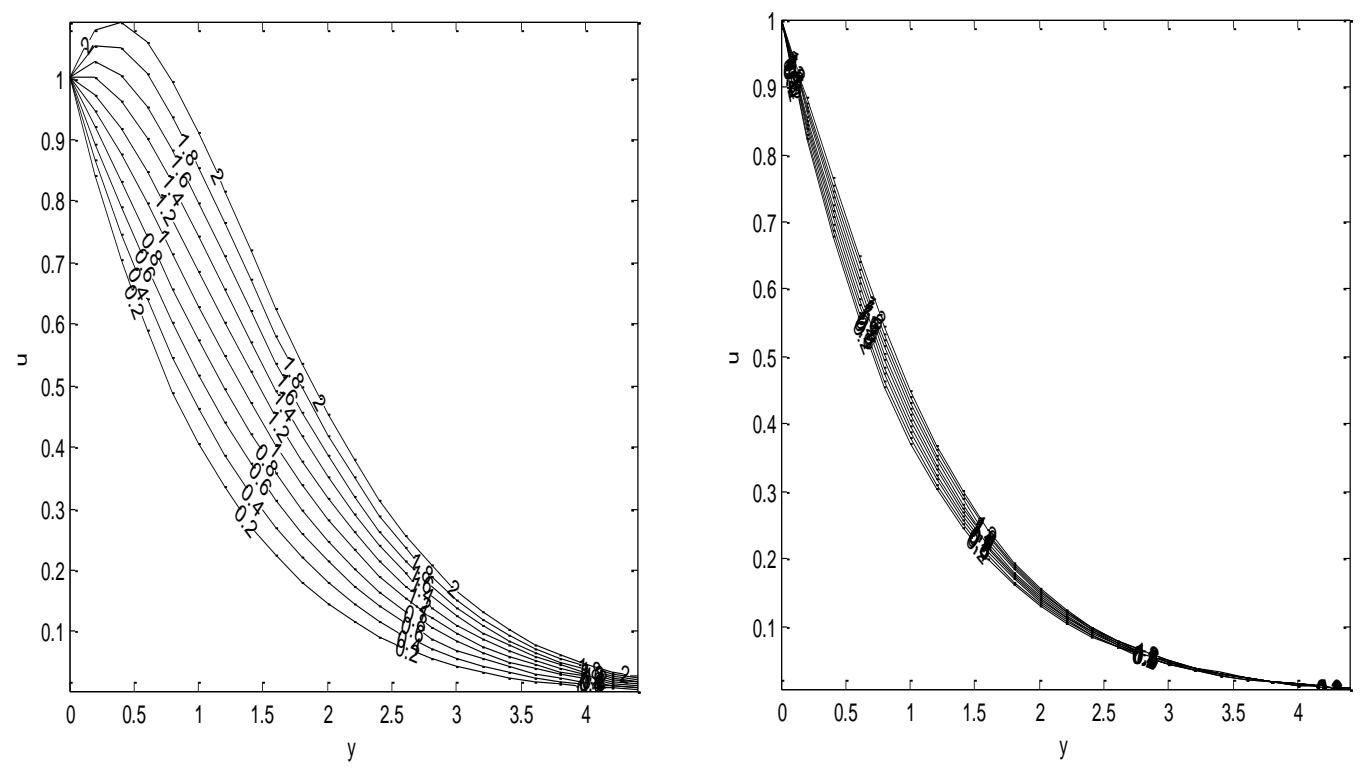

Figure 3. Velocity profile showing the effect of $G r$ for $\operatorname{Pr}=0.71$ and $\operatorname{Pr}=7.0$ respectively, with fixed values of

$$
\left(t=1.0, m_{0}=0.5\right)
$$

Figure 4 displays the effect of magnetic field parameter and $t$ on the skin-friction. It is observed that, skin friction declines as the Hartmann number rises for both Prandtl numbers. This is physically true since increasing magnetic field strength decreases fluid velocity which in turn decreases skin-friction at the wall. Similar results have been found by [19-24]. Although this has been wrongly presented in Table 2 of Sacheti et al. [2] that skin-friction increases with increase in Hartmann number. The above correction will help design engineers and serve as improvement on unsteady flow formation in a vertical channel with transversely applied magnetic field. Figure 5 depicts the role of buoyancy parameter and time on skin friction. It is found that, skin friction increases as buoyancy force increases regardless of the working fluid. Finally, a comparison was made between the present work and the work of [2], it is detected that, the numerical results obtained for the velocity is found to be in excellent agreement as shown in Table 1 while there is discrepancy in the skin friction which has been addressed in Table 2. 

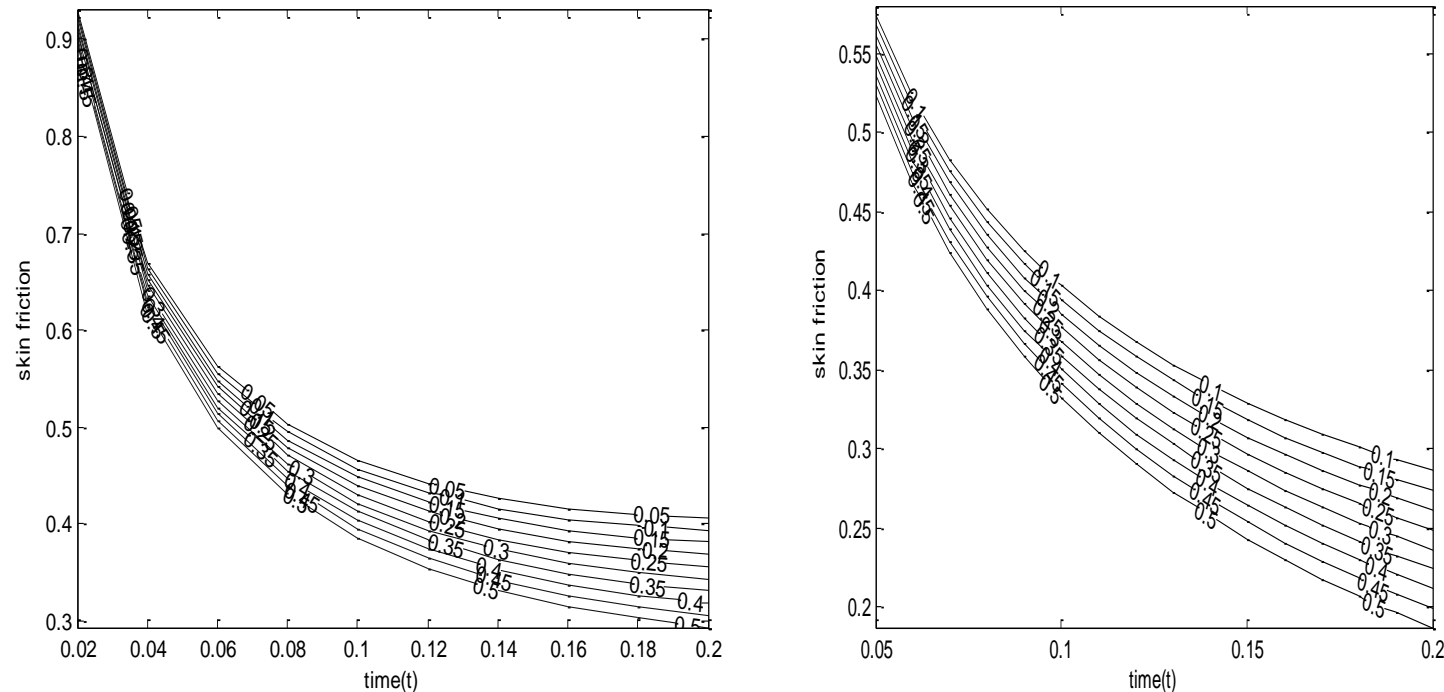

Figure 4. Variation of skin friction for different values of $m_{0}$ when $P r=0.71$ and $\operatorname{Pr}=7.0$ " respectively, with fixed value of $G r=1.0$
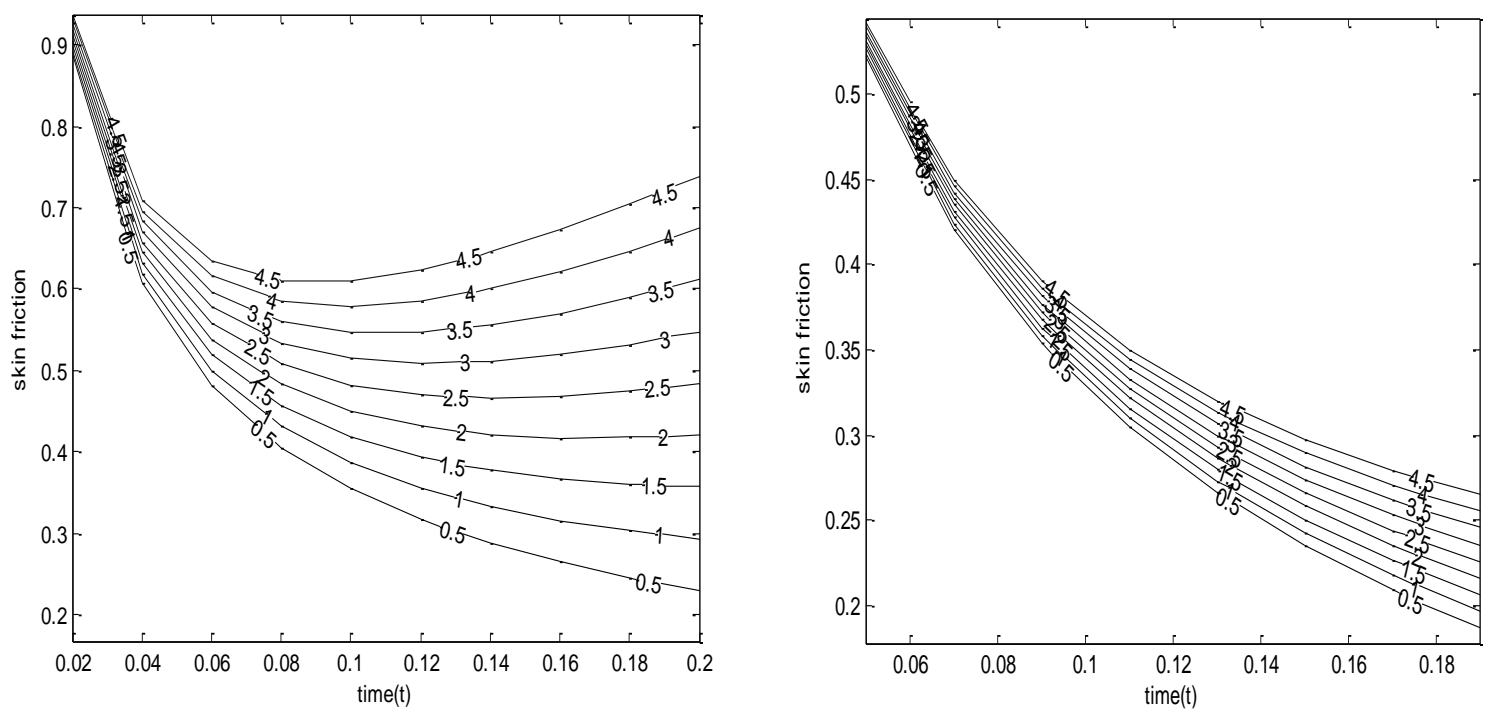

Figure 5. Variation of skin friction for different values of $G r$ when $\operatorname{Pr}=0.71$ and $\operatorname{Pr}=7.0$ respectively, with fixed value of $m_{0}=0.5$

Table 1. Comparison of the numerical values of the velocity obtained using the Riemann-sum approximation method and that obtained using the convolution integration method by [2]

\begin{tabular}{|c|c|c|c|c|c|c|c|c|c|c|c|}
\hline $\boldsymbol{P}$ & $\boldsymbol{G}$ & $y$ & $m_{0}$ & Sacheti et al. [2] & Present work & $P$ & $\boldsymbol{G}$ & $y$ & $m_{0}$ & Sacheti et al. [2] & Present work \\
\hline \multirow[t]{12}{*}{2.0} & 1.0 & 0.5 & 1.0 & 0.7761 & 0.7761 & 7.0 & 1.0 & 0.5 & 1.0 & 0.7236 & 0.7236 \\
\hline & & & 2.0 & 0.7586 & 0.7585 & & & & 2.0 & 0.7071 & 0.7071 \\
\hline & & & 3.0 & 0.7419 & 0.7419 & & & & 3.0 & 0.6913 & 0.6912 \\
\hline & & 1.0 & 1.0 & 0.5202 & 0.5202 & & & 1.0 & 1.0 & 0.4703 & 0.4703 \\
\hline & & & 2.0 & 0.5002 & 0.5003 & & & & 2.0 & 0.4517 & 0.4517 \\
\hline & & & 3.0 & 0.4818 & 0.4818 & & & & 3.0 & 0.4341 & 0.4341 \\
\hline & 3.0 & 0.5 & 1.0 & 0.9149 & 0.9149 & & 3.0 & 0.5 & 1.0 & 0.7576 & 0.7576 \\
\hline & & & 2.0 & 0.8951 & 0.8951 & & & & 2.0 & 0.7405 & 0.7405 \\
\hline & & & 3.0 & 0.8762 & 0.8762 & & & & 3.0 & 0.7242 & 0.7242 \\
\hline & & 1.0 & 1.0 & 0.6402 & 0.6402 & & & 1.0 & 1.0 & 0.4906 & 0.4905 \\
\hline & & & 2.0 & 0.6178 & 0.6177 & & & & 2.0 & 0.4716 & 0.4716 \\
\hline & & & 3.0 & 0.5965 & 0.5965 & & & & 3.0 & 0.4535 & 0.4535 \\
\hline
\end{tabular}


Table 2. Comparison of the numerical values of the skin friction $(\tau)$ obtained using the Riemann-sum

\begin{tabular}{cccccccccc}
\multicolumn{6}{c}{ approximation method and that obtained using the convolution integrals method by [2]. } \\
\hline$G r$ & $m_{0}$ & $t$ & Sacheti et al. $[2]$ & Present work & $G r$ & $m_{0}$ & $t$ & Sacheti et al. $[2]$ & Present work \\
\hline 1.0 & 0.0 & 0.1 & 1.8256 & 0.4215 & 2.0 & 0.0 & 0.1 & 1.8671 & 0.4318 \\
& & 0.2 & 1.3445 & 0.3114 & & & 0.2 & 1.4274 & 0.3222 \\
& 0.3 & 1.1545 & 0.2685 & & & 0.3 & 1.2789 & 0.2996 \\
& 0.4 & 1.0579 & 0.2470 & & & 0.4 & 1.2238 & 0.2885 \\
& 0.1 & 1.9704 & 0.2459 & & 1.0 & 0.1 & 1.9811 & 0.2562 \\
& 0.2 & 1.5281 & 0.0668 & & & 0.2 & 1.5505 & 0.0873 \\
& 0.3 & 1.3593 & -0.2958 & & & 0.3 & 1.3941 & 0.0038 \\
& 0.4 & 1.5786 & -0.0890 & & 0.4 & 1.3230 & -0.0486 \\
2.0 & 0.1 & 2.1407 & 0.0758 & & & 0.1 & 2.1519 & 0.0861 \\
& 0.2 & 1.7591 & 0.1628 & & 0.2 & 1.7830 & -0.1426 \\
& 0.3 & 1.6315 & -0.2958 & & & 0.3 & 1.6700 & -0.2659 \\
& 0.4 & 1.5786 & -0.3870 & & & 0.4 & 1.633 & -0.3476 \\
3.0 & 0.1 & 2.3057 & -0.0889 & & 3.0 & 0.1 & 2.3173 & -0.2385 \\
& 0.2 & 1.9767 & -0.3790 & & & 0.2 & 2.0042 & -0.5637 \\
& 0.3 & 1.8819 & -0.5430 & & 0.3 & 1.9242 & -0.7427 \\
& 0.4 & 1.8520 & -0.6548 & & 0.4 & 1.9135 & -0.8602 \\
\hline
\end{tabular}

\section{CONCLUSION}

In this present work, a scrutiny of the impulsive motion of an electrically conducting fluid near an infinite vertical plate has been done. The governing equations have been solved by combining the LT technique to transform the PDE to ODE, while the RSA approach is employed to transform the closed-form solutions from Laplace domain to time domain. From this investigation, we observed that, the velocity obtained by the current technique are in precise agreement with those described by Sacheti et al in Table 1 while there is discrepancy in the skin friction with the result of published work of Sacheti et al. which has been addressed in Table 2. Results show that less computational time is incurred by using the Riemann-sum approximation approach.

\section{REFERENCES}

[1] V. M. Soundalgekar and M. R. Patil, "Stokes problem for infinite vertical plate with constant heat flux," Atrophy. Space Sci, vol. 70, pp. 179-182, 1980.

[2] N. C. Sacheti, P. Chandran, A. K. Singh, "An exact solution for unsteady magnetohydrodynamic free convection flow with constant heat flux," Int. Comm. In Heat and Mass Transfer, vol. 21, no. 1, pp. 131-142, 1994.

[3] N. G. Kafousias, N. D. Nanousis and G. A. Georgantopoulos, "Free convection effects on the stokes problem for an infinite vertical limiting surface with constant suction," Astrophys. Space Sci, vol. 64, pp. 391-399, 1979.

[4] F. S. Ibrahim, A. M. Elaiw and A. A. Bakr, "Effect of chemical reaction and radiation absorption on the unsteady MHD free convection flow past a semi infinite vertical Permeable Moving Plate with Heat Source and Suction" Communication in Nonlinear Science and Numerical Simulation, vol. 13, no. 6, pp. 1056-1066, Aug. 2008.

[5] A. Rao, S. Shivaiah and Sk. Nuslin, "Radiation effect on an unsteady mhd free convective flow past a vertical porous plate in the presence of soret" Adv. In App. Sci. Res, vol. 3, pp. 1663-1676, 2012.

[6] A. Khalid, I. Khan, A. Khan and S. Shafie, "Unsteady MHD free convection flow of cassion fluid past over an oscillating vertical plate embedded in a porous medium," Engineering Science and Technology, an Int. Journal, vol. 18, 309-317, 2015.

[7] C Israel-Cookey, A Ogulu and V. B Omubo-Pepple, "Influence of viscous dissipation and radiation on unsteady MHD free convection flow past an infinite heated vertical plate in a porous medium with time-dependent suction," Int. Journal of Heat and Mass Transfer, vol. 46, no. 13, pp. 2305-2311, Jun. 2003.

[8] A. K. Singh, "MHD free convection flow past an accelerated vertical porous plate in a rotating fluid," Astrophys. Space Sci., vol. 103, pp. 155-163, 1984.

[9] A. K Singh, "Unsteady free convection flow through a rotating porous medium," Indian National Sci. Acad, vol. 52, no. 3A, pp. 647-652, Jan. 1986.

[10] N. D. Nanousis, G. A. Georgantopoulos and A. I. Papaioannou, "Hydromagnetic free convection flow in the stokes problem for a porous vertical limiting surface with constant suction," Atrophy. Space Sci, vol. 70, pp. 377-3831980.

[11] C.K. Chen and H.C. Weng, "Natural convection flow in a vertical microchannel," J Heat Transf., vol. 127, no.9, pp. 1053-1059, Sep.2015.

[12] S.S. Chawla, "Magnetohydrodynamics unsteady free convection", ZAMM 1967;47:499-508.

[13] B. K. Jha, B. Aina and S. Isa, "Transient magnetohydrodynamic free convective flow in vertical micro-concentricannuli," Proc IMechE Part N: J Nanoengineering and Nanosystems, Apr. 2015, doi: 10.1177/1740349915578956.

[14] B. K. Jha, B. Aina and S. Isa, "Fully developed MHD natural convection flow in a vertical annular microchannel: An exact solution", Journal of King Saud university-Science, vol. 27, no. 3, pp. 253-259, Jul. 2015.

[15] B. K. Jha and B. Aina, "MHD natural convection flow in a vertical micro-porous- annulus in the presence of radial magnetic field," Journal of Nanofluids, vol. 5, no. 2, Oct. 2015, doi: 10.1166/jon.2016.1204. 
[16] R. Ellahi, M. Khan and N. A. Shah "Combine porous and magnetic effect on some fundamental motions of Newtonian fluids over an infinite plate," Journal of Porous Media, vol. 21, no. 7, pp. 589-605, 2018.

[17] B. K. Jha, M. O. Oni, "Transient natural convection flow between vertical concentric cylinders heated/cooled asymmetrically," Proc IMechE Part A: J Power and Energy, vol. 232, no. 7, Feb. 2018.

[18] D. Y. Tzou, "Macro to microscale heat transfer: the lagging behaviour," Taylor and Francis, London, pp. 576, Nov. 2014.

[19] B. K. Jha, M. O. Oni, "Role of magnetic field on mixed convective flow between two concentric microtubes with heat generation/absorption: An exact solution," Proc IMechE Part N: J Nanoengineering and Nanosystems, May 2018, doi: 10.1177/2397791418776520.

[20] M. Narahari and L. Debnath, "Unsteady magnetohydrodynamic free convection flow past an accelerated vertical plate." ZAMM Z. Angew. Math. Mech., Jan. 2013.

[21] B. K. Jha, M. O. Oni, "Mathematical modelling of combined pressure driven and electrokinetic effect in a channel with induced magnetic field: An exact solution," Journal of King Saud University-Science, vol. 31, no. 4, pp. 575585, Oct 2019, doi: https://doi.org/10.1016/j.jksus.2018.10.009.

[22] A.K. Singh, "MHD free convection flow in stokes problem for a porous vertical plate," Astrophys Space Sci, vol. 87, no. 455, 1982.

[23] P. Chandran, N. C. Sacheti and A. K. Singh, "Unsteady hydromagnetic free convection flow with heat flux and accelerated boundary motion," Journal of the Physical Society of Japan, vol. 67, pp. 124-129, 1989.

[24] P. Chandran, N. C. Sacheti and A. K. Singh, "A unified approach to analytical solution of a hydromagnetic free convection flow," Scientiae mathematicae Jpn, vol. 53, no. 467, 2001.

\section{BIOGRAPHIES OF AUTHORS}

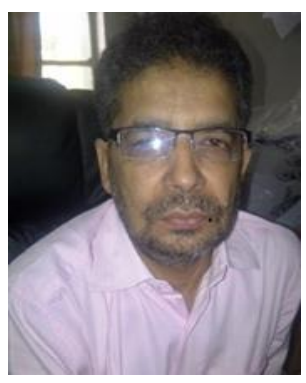

Basant K. Jha was born in Darbhanga, Bihar, India. He is a professor in Applied Mathematics at Ahmadu Bello University, Zaria, Nigeria. Prof. Jha has published more than 160 papers in reputed National/International journals. His research interest includes flow through porous media, magneto hydrodynamics, computational fluid dynamics, and heat and mass transfer.

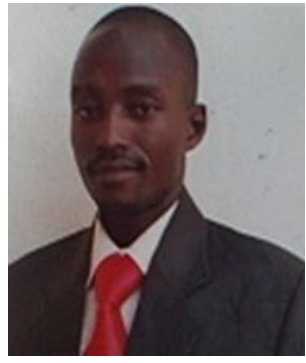

Luqman A. Azeez was born in Okeho, Oyo State, Nigeria. He is a Lecturer Federal College of Education, Zaria, Nigeria. He had his B.Sc. in the Department of Mathematics, ABU Zaria (2012) and his MSc. In (2017). His areas of interest include magneto hydrodynamics, computational fluid dynamics, and heat and mass transfer, channel.

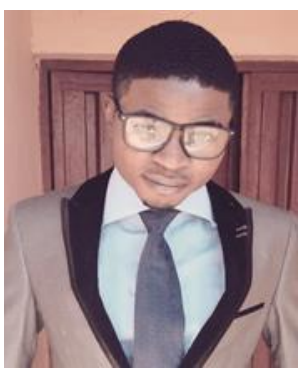

Michael O. Oni was born in Zaria, Kaduna State, Nigeria. He is a Lecturer and currently a $\mathrm{PhD}$ Student in the Department of Mathematics Ahmadu Bello University (ABU), Zaria, Nigeria. He had his B.Sc. (First Class Honours) in the Department of Mathematics, ABU Zaria (2012) and his MSc. In (2016). He immediately started his $\mathrm{PhD}$ in fluid mechanics (electroosmotic flow) in 2016 and almost rounding up. M.O. Oni has published more than 20 papers in reputed National/International journals. His areas of interest include flow through porous media, magneto hydrodynamics, electroosmotic flow, computational fluid dynamics, and heat and mass transfer, micro-annulus. 